\title{
Self-Consistent Analysis of the Relative Intensity Noise Characteristics in the Strained AlGaInN Laser Diodes with the High Frequency Current Modulation Effects
}

\author{
Jong Chang Yi and Hyung Uk Cho \\ Electronic Eng. Department, Hongik University, Sangsu 72-1, Mapo, Seoul 121-791, Korea \\ Young Min Jhon* \\ Korea Institute of Science and Technology, Hawolgok 39-1, Seongbuk, Seoul 136-791, Korea
}

(Received February 11, 2008 : revised March 14, 2008)

\begin{abstract}
The relative intensity noise (RIN) characteristics in $405 \mathrm{~nm}$ blue laser diodes grown on wurtzite AlGaInN multiple quantum well structures were investigated using the rate equations with the quantum Langevin noise model. The device parameters were extracted from the optical gain properties of the MQW active region using the self-consistent numerical method developed for calculating the multiband Hamiltonian in the strained wurtzite crystal. These methods have been applied to laser diodes for various conditions including the external feedback and the high frequency current injection.
\end{abstract}

OCIS codes : 140.5960, 270.2500, 130.0250, 210.0210, 140.2020

\section{INTRODUCTION}

It is well known that the GaN-based semiconductors are the best material for short wavelength devices. Especially, the quaternary alloy AlGaInN has wide band gap energy, varying with the composition and almost covering the visible spectrum while extending to the ultraviolet region. So, technologies of nitride semiconductors have led to the development of electrical devices for applications of high power and high frequency such as optical data storage and mobile communication. In these applications, the blue laser diodes have become important building blocks for the next-generation optical data storage elements and optical recording systems. However, their performance sometimes lags behind the demand required for a fast data access time and operation speed. Such limitations are imposed in part by the noises of the laser diodes used in the optical pick-ups [1]. In this paper, characteristics of such an intensity noise, known as the relative intensity noise (RIN), were analyzed for nitride compound semiconductor laser diodes. The primary source of the intensity noise in the semiconductor laser diode is the spontaneous emission that is usually amplified due to the external feedback from the optical disks. Such quantum noise has been modeled using the quantum Langevin formalism [2]. The required material parameters have been extracted by using the self-consistent Poisson-Schrödinger and the multiband Hamiltonian for strained wurtzite crystals [3,4]. To show the validity of this method, the simulation results were compared to the analytic solutions when the external feedback effect is ignored, which shows a good agreement. If the optical utilization efficiency is too high, however, relative intensity noise (RIN) generated by the blue-violet laser diode during play will interfere with play. This problem is being avoided by using different optical utilization efficiencies for play and record. One of the factors behind RIN is quantum noise, so that RIN increases as laser diode optical output decreases. In general, reducing the optical output of a blue-violet laser diode to low power will cause RIN to exceed the $-125 \mathrm{~dB} / \mathrm{Hz}$ level accepted as the limit for disc play. This would push RIN over $-125 \mathrm{~dB} / \mathrm{Hz}$, making playback difficult. So, one way to reduce the external feedback effect is to modulate the injection current to the laser cavity up to a certain high frequency $[1,3]$. Although the technique of high-frequency injection (HFI) can solve this problem, the proper modulation frequency must be chosen practically. In this work, the optimum conditions for the high frequency current injection noise 
reduction method on the RIN characteristics has been investigated to reduce the external feedback effect that is inevitable in DVD-ROM or RW pick-up systems.

\section{SELF-CONSISTENT ANALYSIS OF THE STRAINED BLUE LASER DIODE}

The $405 \mathrm{~nm}$ laser diode under investigation has typical AlGaInN MQW active structures as shown in Figure 1. The quantum well regions are $3 \mathrm{~nm} \mathrm{In}_{0.12} \mathrm{Ga}_{0.88} \mathrm{~N}$ and the barrier regions are $\mathrm{In}_{0.02} \mathrm{Ga}_{0.98} \mathrm{~N}$. The $\mathrm{SCH}$ regions are $\mathrm{Al}_{0.2} \mathrm{Ga}_{0.8} \mathrm{~N}$ and the cladding regions are $\mathrm{N}$ - and $\mathrm{P}-$ doped $\mathrm{Al}_{0.13} \mathrm{Ga}_{0.87} \mathrm{~N}$ sandwiched by GaN ohmic contact layers $[5,6]$.

Figure 2 (a) shows the energy band diagram of multiple quantum well (MQW) and electron blocking layer (EBL) and (b) shows E-k band diagram and Density of States (D.O.S.) of conduction and valence band. For the enhanced optical performance, P-doped $\mathrm{Al}_{0.3} \mathrm{Ga}_{0.7} \mathrm{~N}$ $\mathrm{EBL}$ is indispensable for the suppression of electron overflow from the active layer to the p-type cladding layer. The gain and spontaneous emission spectra of the AlGaInN 3 QW LD are obtained by numerical analysis of self-consistent Poisson equations and the Hamiltonian equations for multiband strained wurtzite multiple quantum well structures [7]. Figure 3 (a) and (b) show the variation of the optical gain properties due to the strain effects. The compressive strain effect led to a $5 \mathrm{~nm}$ blue shift in the emission spectra and enhanced optical performance such as gain and differential gain. The strain effects due to the lattice constant smaller than GaN in the buffer layer affects the differential gain. Figure 4 (a) and (b) show the calculated gain spectra and the spontaneous emission spectra from the AlGaInN 3 quantum well structures when the injection carrier density varies from $1.8 \times 10^{19} / \mathrm{cm}^{3}$ to $5.0 \times 10^{19} /$ $\mathrm{cm}^{3}$ by the step of $1.6 \times 10^{12} / \mathrm{cm}^{3}$. All relevant simulation parameters, such as the transition energy $E_{T}$, the Fermi energy separation versus the injection carrier concentration $E_{q f p}$, the population inversion parameter $n_{s p}$, the transparency carrier concentration $N_{t r}$, the maxi-

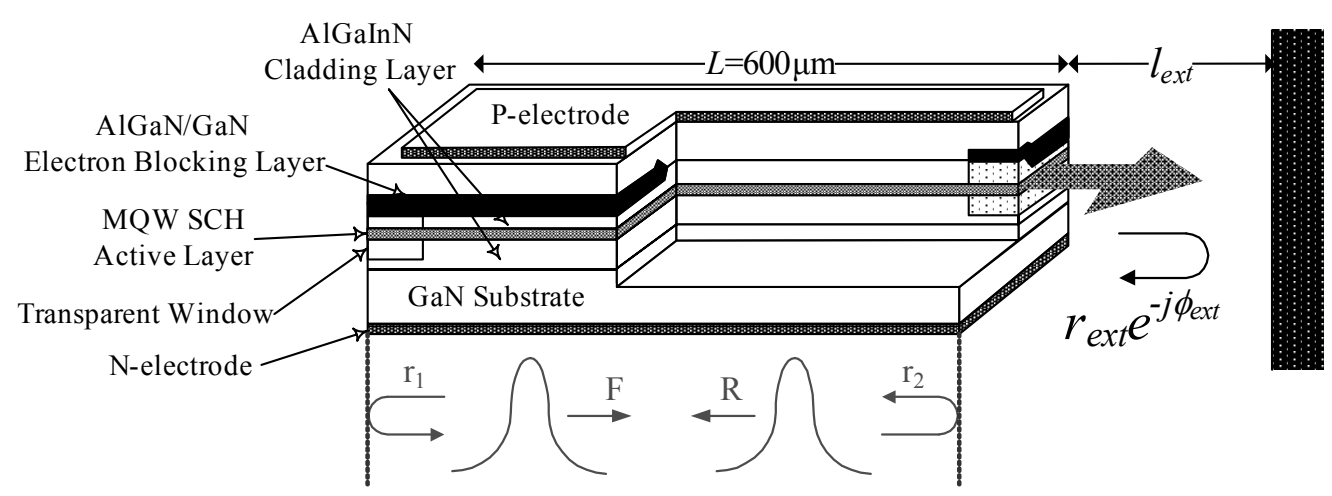

FIG. 1. Schematic diagram of the AlGaInN MQW laser diode structure with external feedback effects.

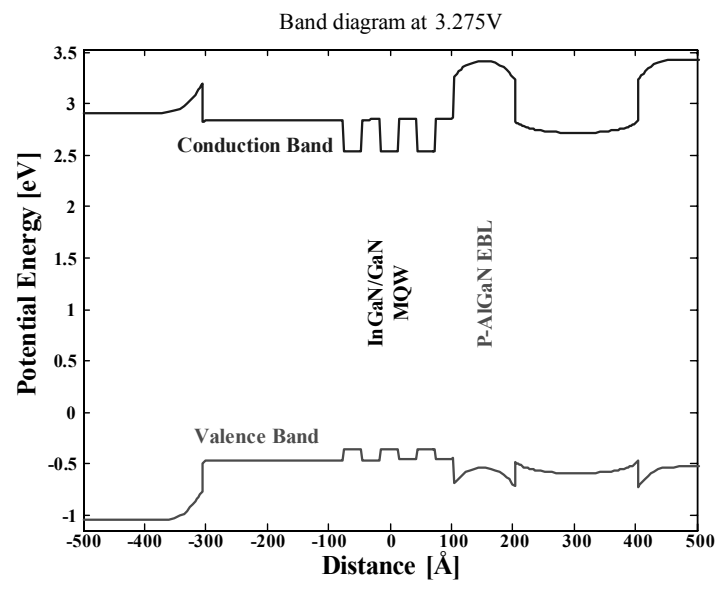

(a)
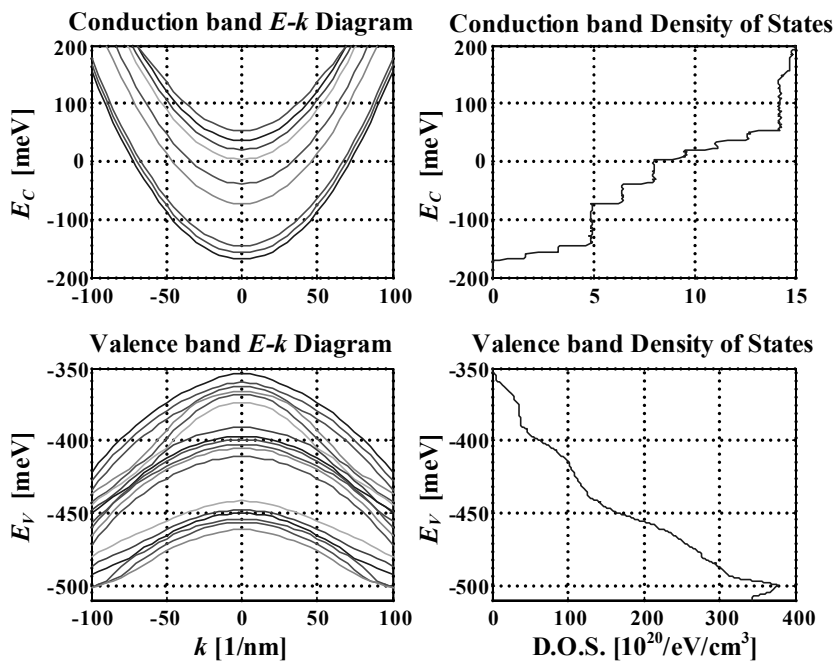

Valence band Density of States

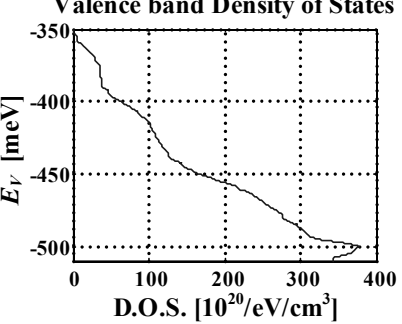

(b)

FIG. 2. (a) Band diagram of MQW \& EBL region. (b) E-k diagram and D.O.S of conduction and valence band. 


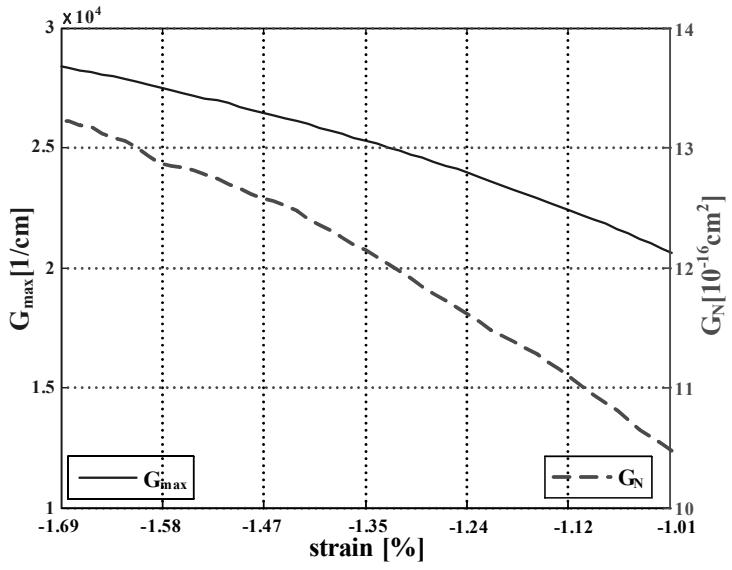

(a)

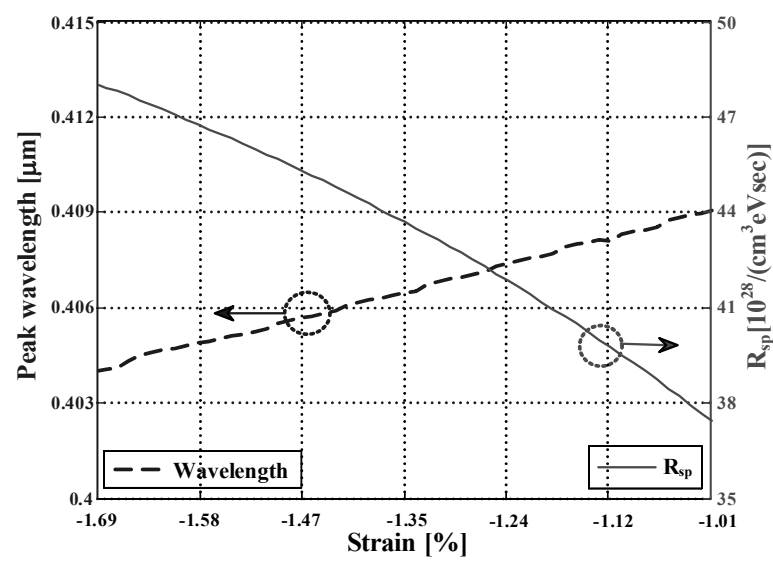

(b)

FIG. 3. (a) The variation of maximum material gain $\left(G_{\max }\right)$ the differential gain $\left(G_{N}\right)$ due to compressive strain effects. (b) The variation due to compressive strain effects on maximum of spontaneous emission ratio $\left(R_{s p}\right)$ and peak wavelength.

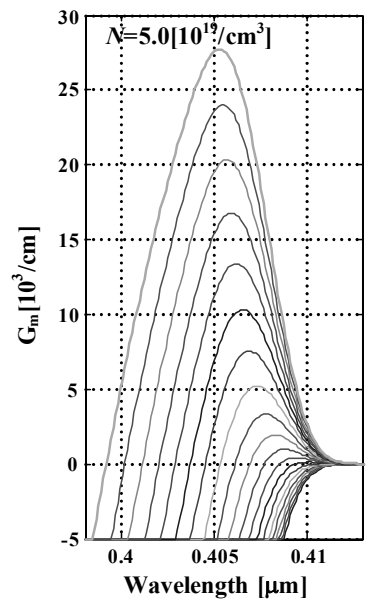

(a)

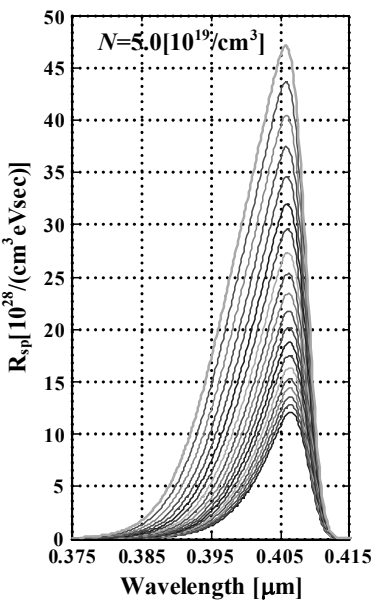

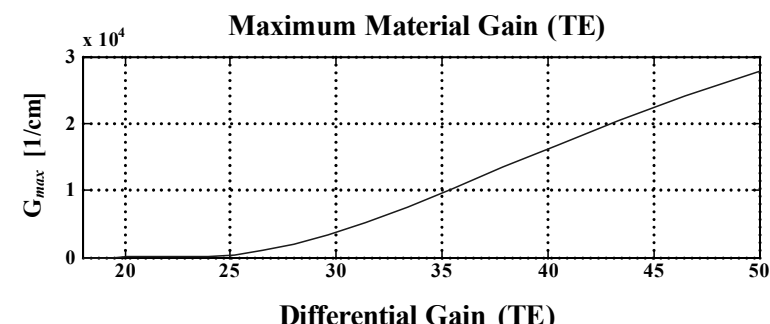

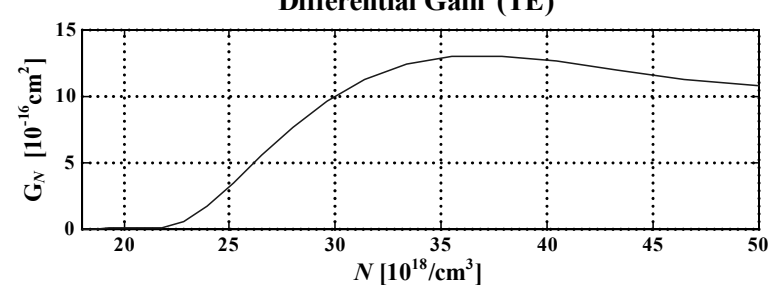

(b)

FIG. 4. (a) Gain $\left(G_{m}\right)$ and Spontaneous emission $\left(R_{s p}\right)$ spectra for the various carrier injections from $1.8 \times 10^{19}\left[\mathrm{~cm}^{-3}\right]$ to $5.0 \times 10^{19}\left[\mathrm{~cm}^{-3}\right]$ in 20 steps. (b) Maximum material gain $\left(G_{\max }\right)$ \& Differential Gain $\left(G_{N}\right)$ of AlGalnN MQW structures for the strained wurtzite crystals.

mum material gain $G_{m a x}$, and the differential gain $G_{N}$, were extracted from the gain calculation.

\section{RELATIVE INTENSITY NOISE ANALYSIS OF LASER DIODE}

The quantum noise due to the external feedback from the optical disc surface into the laser cavity is modeled as shown in Fig. 1. F and $\mathrm{R}$ shown in the figure denote the forward and reverse traveling optical fields, $r_{\text {ext }}, \phi_{\text {ext }}, l_{\text {ext }}$ the reflection coefficient, phase retardation, and the length of the external cavity, respectively. The quantum noise has been modeled with the quantum Langevin formalism and the required material parameters have been extracted by using the selfconsistent Poisson and the multiband Hamiltonian. The relative intensity noise (RIN) of the laser diode is defined as the ratio of the laser intensity noise $\delta P(t)$ to the average laser power $P_{0}(t)$, or

$$
R I N=\frac{S_{P}(\omega)}{P^{2}}=\frac{\int_{-\infty}^{\infty} d \tau\langle\delta P(t+\tau) \delta P(t)\rangle e^{-i \omega \tau}}{P^{2}}
$$

The characteristics of the laser intensity noise can be obtained by analyzing the rate equations:

$$
\begin{aligned}
& \frac{d}{d t} N(t)=\frac{I(t)}{q}-\frac{N(t)}{\tau_{e}}-G(t) P(t)+F_{N}(t) \\
& \frac{d}{d t} P(t)=(G(t)-\gamma) P(t)+R_{s p}+2 \chi P_{f b}+F_{P}(t) \\
& \frac{d}{d t} \phi(t)=\frac{1}{2} \alpha(G(t)-\gamma)-\frac{n_{m}}{n_{g}}(\omega-\Omega)+\chi \phi_{f b}+F_{\phi}(t)
\end{aligned}
$$


with

$$
\begin{aligned}
P_{f b} & =\sqrt{P(t) P(t-\tau)} \cos [\omega \tau+\phi(t)-\phi(t-\tau)] \\
\phi_{f b} & =\sqrt{\frac{P(t-\tau)}{P(t)}} \sin [\omega \tau+\phi(t)-\phi(t-\tau)]
\end{aligned}
$$

where $N(t), P(t), \phi(t)$ and $I(t)$ denotes the carrier density, the photon density, the instantaneous phase and current in the cavity of LD. $G(t)$ is the optical gain, $R_{s p}$ is the spontaneous emission rate, $\gamma$ is the optical loss, $\alpha$ is linewidth enhancement factor, $n_{m}$ is mode refractive index, $n_{g}$ is the group refractive index, $F_{i}$ is the Langevin noise factors [8], $\chi$ is the external feedback coefficient, and $\tau$ is the round trip time for the external cavity, respectively [9]. Random fluctuations of the lasing frequency are caused by carrier density noise and by the phase noise. The other parameters follow conventional notations. The Langevin noise functions show characteristic behaviors as following:

$$
\begin{array}{cl}
\left\langle F_{i}(t)\right\rangle \quad & 0 \quad \text { for } i=P, N, \phi \\
\left\langle F_{i}(t) F_{j}\left(t^{\prime}\right)\right\rangle= & 2 D_{i j} \delta\left(t-t^{\prime}\right) \quad \text { for } j=P, N, \phi \\
& 2 D_{p p}=2 n_{s p} \frac{\bar{P}}{\tau_{P}} \\
2 D_{N N}=\left(2 n_{s p}-1\right) \frac{\bar{P}}{\tau_{P}}+\frac{\bar{N}}{\tau_{e}} & \\
2 D_{P N}=-\left(2 n_{s p}-1\right) \frac{\bar{P}}{\tau_{P}} & \\
2 D_{\phi \phi}=n_{s p} \frac{1}{2 \bar{P} \tau_{P}} &
\end{array}
$$

Here, $\bar{N}, \bar{P}$ are the averages of $N, P$ in the steady state, $\tau_{P}$ is the photon lifetime, $\tau_{e}$ is the electron lifetime and $n_{s p}$ is the population inversion factor [8]. The optical gain in (2) can be expressed in terms of differential gain as:

$$
G=\frac{\frac{d G}{d N}\left(N-N_{t r}\right)}{1+\varepsilon P} \equiv \frac{G_{N}\left(N-N_{t r}\right)}{1+\varepsilon P}
$$

Here, $G_{N}$ is the differential gain, $N_{t r}$, the transparency carrier concentration, and, $\mathcal{E}$ the gain suppression coefficient.

\section{SIMULATION RESULTS}

In the time domain analysis, the Langevin noise functions were implemented by random number generators with amplitude proportional to the square root of the diffusion coefficients. Figure 5 shows the RIN characteristics of the $405 \mathrm{~nm}$ blue laser diode. This shows the Fourier transformed spectra of the noise terms for various optical output power levels of the laser diode. The numerically obtained RIN spectra show a good agreement with the analytic solutions as shown in dashed lines.

Figure 6 shows the numerical simulation results of the RIN spectra when the external feedback is nonnegligible. This result was compared to $-125 \mathrm{~dB} / \mathrm{Hz}$, which is the RIN requirement of the commercial optical pick-up system. When the external feedback rate is smaller than $0.01 \%$, the RIN decreased drastically as the power of LD increased. When the external feedback rate is over $1 \%$, the RIN characteristics deteriorates beyond use. We can see any external feedback rate larger than $0.1 \%$ would result in the RIN to be higher than $-125 \mathrm{~dB} / \mathrm{Hz}$. These results indicate that the RIN decreases as the laser power increases. However, the low power LD performance plays a key role in the optical pick-up system since the majority of the operation is in the reading mode.

So, one way to reduce the external feedback effect is modulating the injection current to the laser cavity up to a certain frequency $[10,11]$. Figure 7 shows the driving method of a laser diode with a high frequency current superimposition to reduce the RIN. The reference value of the modulation current $\left(\mathrm{I}_{\text {modo }}\right)$ is the margin between the bias current $\left(\mathrm{I}_{\text {Bias }}\right)$ and the threshold current $\left(\mathrm{I}_{t h}\right)$. It is worth to note that when the injection current becomes smaller than the threshold by a large modulation depth, i.e., $\mathrm{I}_{\text {mod }}-\mathrm{I}_{\text {Bias }}>\mathrm{I}_{\text {Bias }}-\mathrm{I}_{\text {th }}$, the RIN characteristics deteriorate significantly. Figure 8 (a) shows the low frequency RIN characteristics at the injection current bias levels for $10 \mathrm{~mW}$ with different modulation depths. Fig. 8 (a) shows the RIN result when the modulation depth is $1 \mathrm{I}_{\text {modo }}$, while Fig. 8 (b) when

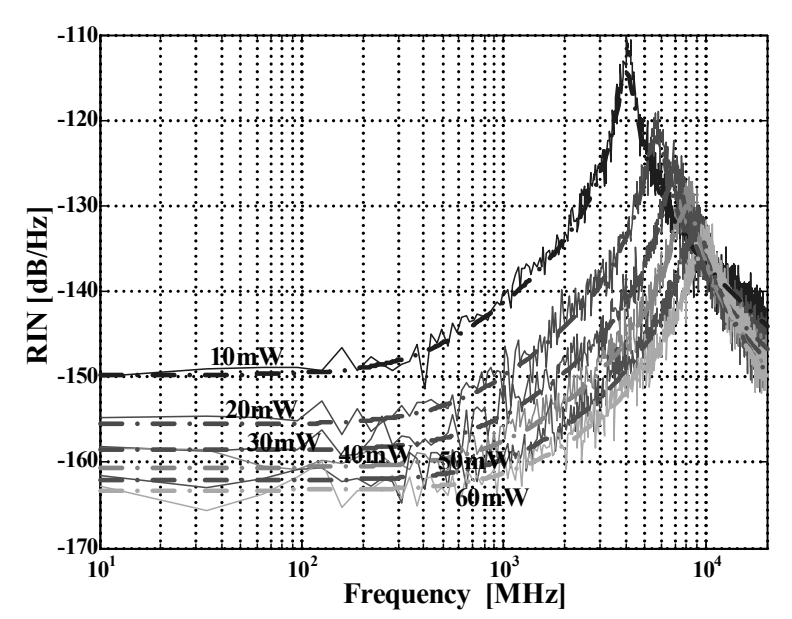

FIG. 5. The Relative intensity noise (RIN) spectra without external feedback effects. 


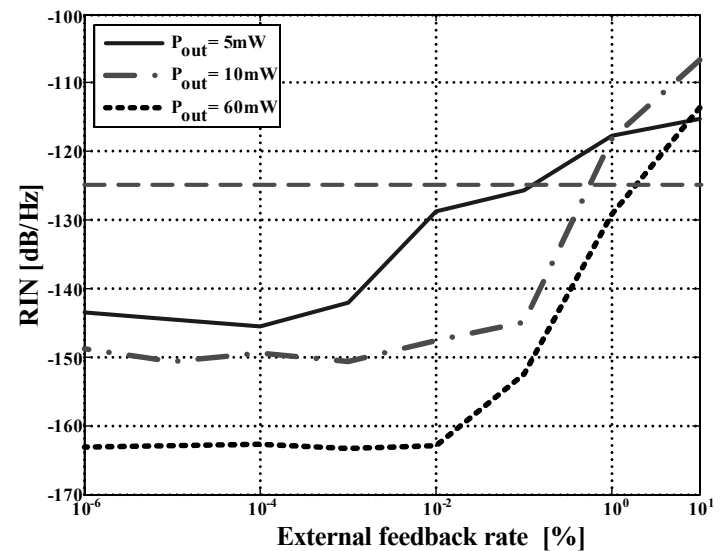

(a)

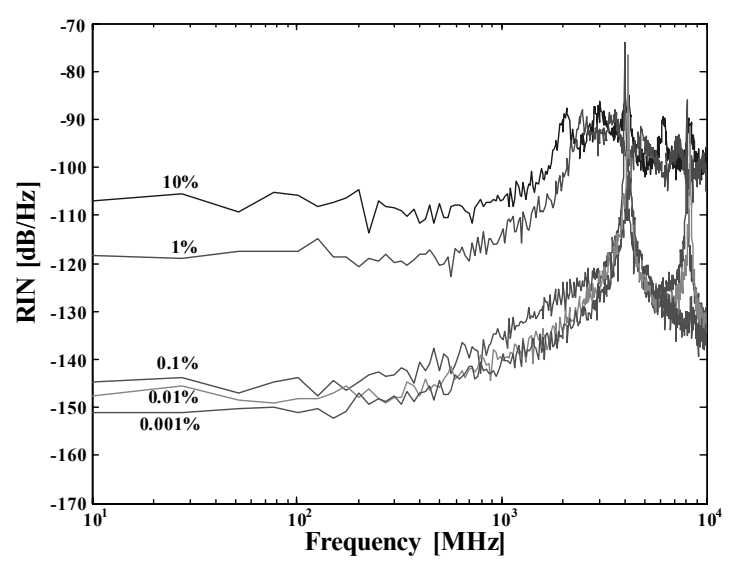

(c)

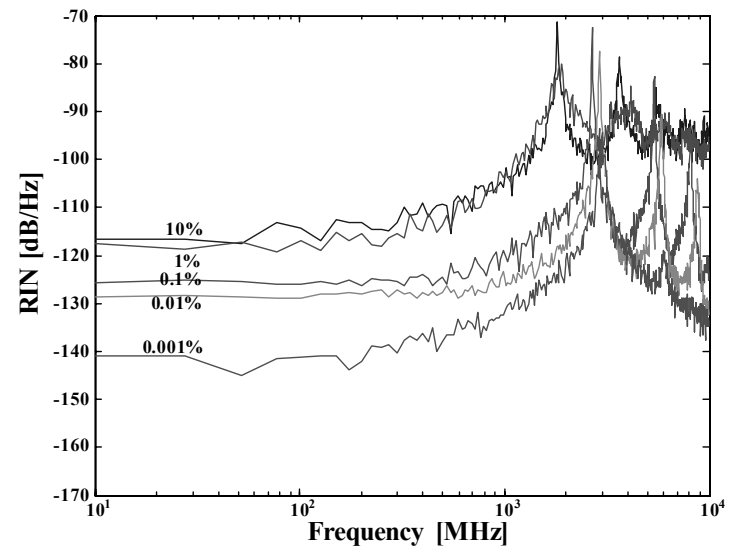

(b)

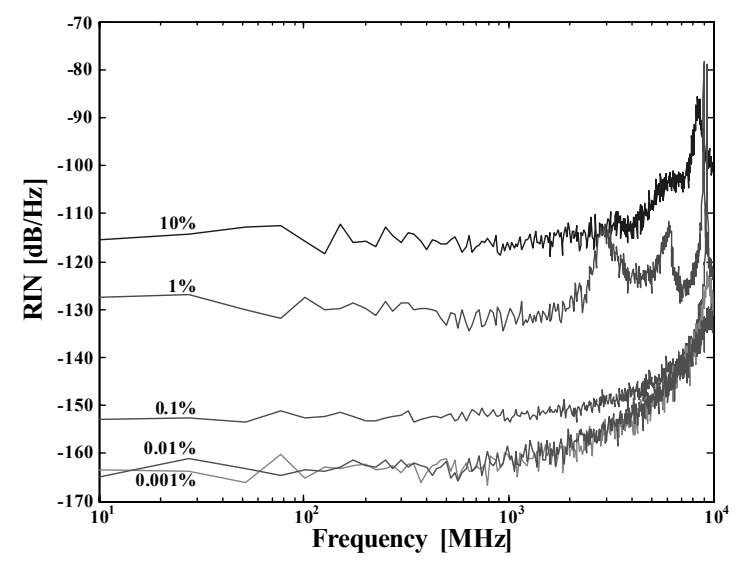

(d)

FIG. 6. (a)The RIN spectra of output power for various external feedback rates. (b) The RIN spectra for $5 \mathrm{~mW}$ output power for various external feedbacks. (c) The RIN spectra for $10 \mathrm{~mW}$ output power for various external feedbacks. (d) The RIN spectra for $60 \mathrm{~mW}$ output power for various external feedbacks.

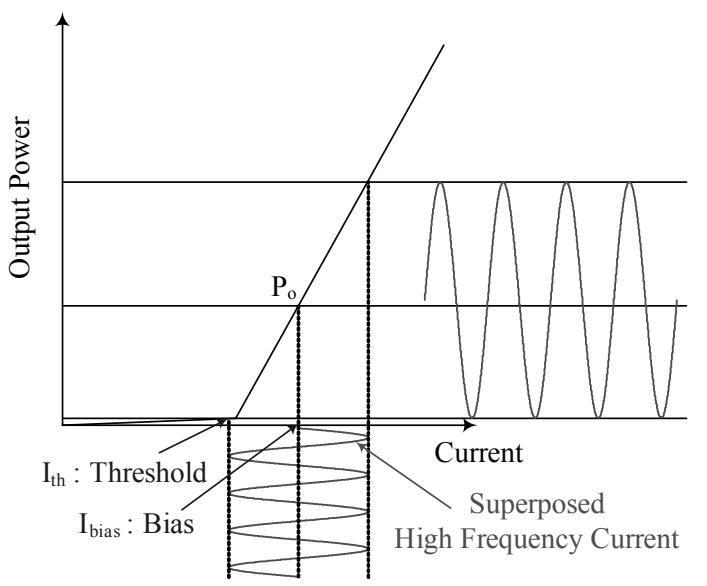

FIG. 7. Driving method of a laser diode with a high frequency current superimposition.

$0.5 \mathrm{I}_{\text {modo }}$. The larger modulation depth gave the RIN characteristics with $1 \sim 2 \mathrm{GHz}$ modulation frequency is above $-125 \mathrm{~dB} / \mathrm{Hz}$, while the smaller modulation depth gave the RIN characteristics at the similar frequency under $-125 \mathrm{~dB} / \mathrm{Hz}$. In this case, despite the fluctuation due to random signal, the optimum modulation frequency turned out to be around $5 \sim 6 \mathrm{GHz}$ and the RIN reduction of more than $8 \mathrm{~dB} / \mathrm{Hz}$ was obtained. Figure 9 shows the RIN characteristics for $5 \mathrm{~mW}$ output power. Figure 9 (a) shows RIN results when the modulation depth is $1 \mathrm{I}_{\text {mod }}$, while Figure 9 (b) when $0.5 \mathrm{I}_{\text {modo }}$. From Fig. 9, one can once again see that it is more favorable to use a smaller modulation depth for a RIN reduction. In this case, a RIN reduction of more than $10 \mathrm{~dB} / \mathrm{Hz}$ could be available by optimizing the injection modulation frequency around $3 \sim 4 \mathrm{GHz}$, and optimizing the modulation depth as well.

\section{CONCLUSIONS}

In this work, the relative intensity noise characteristics in $405 \mathrm{~nm}$ laser diodes grown on wurtzite InAlGaN multiple quantum well structures were investigated using 


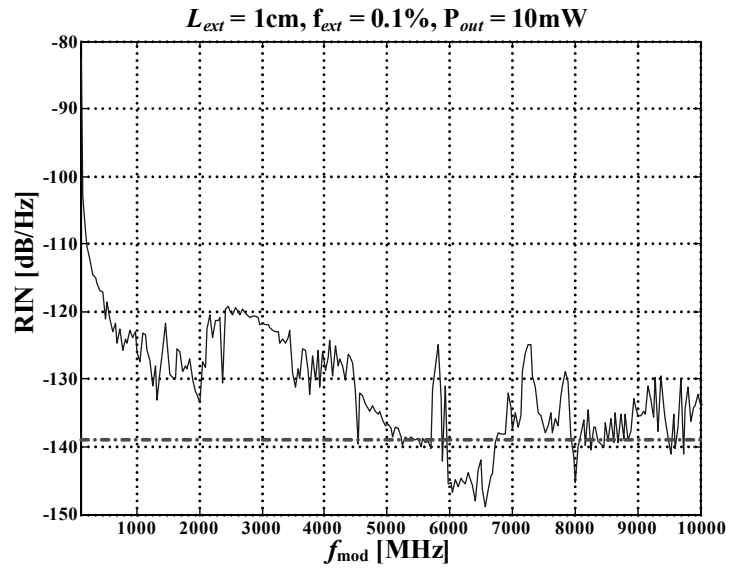

(a)

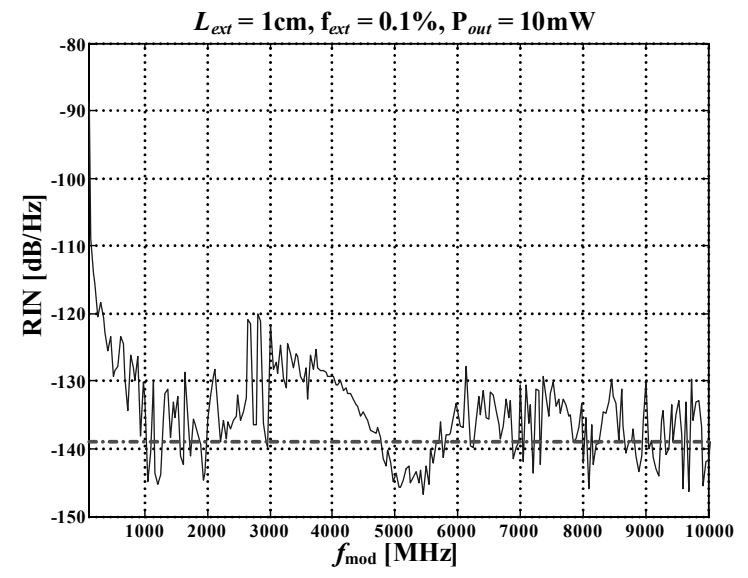

(b)

FIG. 8. (a) The RIN of the once of modulation current $\left(\mathrm{I}_{\text {modo }}\right)$ for the $10 \mathrm{~mW}$ output power. (b) The RIN of the half of modulation current $\left(0.5^{*} \mathrm{I}_{\bmod 0}\right)$ for the $10 \mathrm{~mW}$ output power.

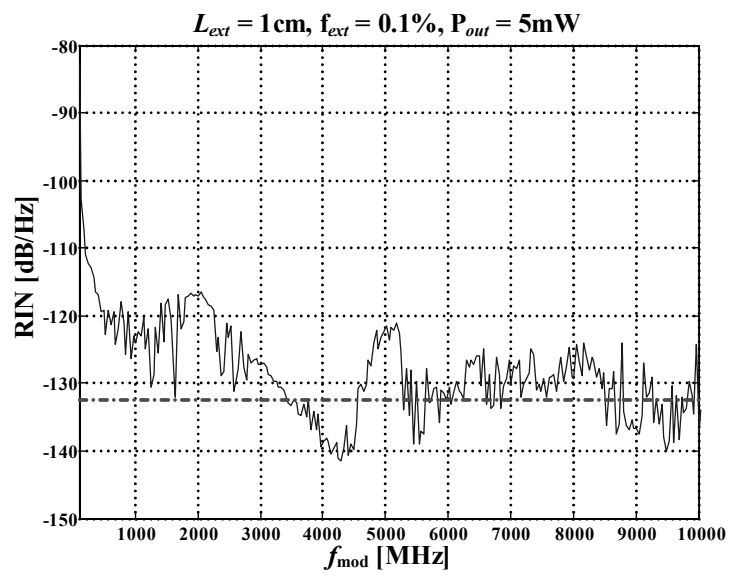

(a)

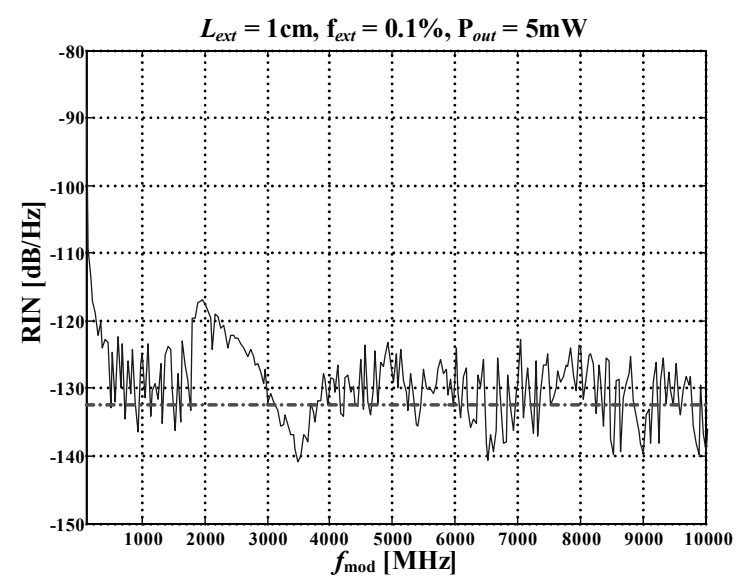

(b)

FIG. 9. (a) The RIN of the once of modulation current ( $\mathrm{I}_{\text {modo }}$ ) for the $5 \mathrm{~mW}$ output power. (b) The RIN of the half of modulation current $\left(0.5 * \mathrm{I}_{\text {modo }}\right)$ for the $5 \mathrm{~mW}$ output power.

the rate equations with the quantum Langevin noise model. The device parameters were extracted from the optical gain properties of the MQW active region using the self-consistent Poisson equation and the multiband Hamiltonian for the strained wurtzite crystal. The simulation results indicate that the critical external feedback for $-125 \mathrm{~dB} / \mathrm{Hz}$ RIN is less than $0.1 \%$ and it becomes more severe as the LD power decreases as in the reading mode. To alleviate the external feedback effect on the RIN characteristics, we proposed a new method by optimizing the modulation depth as well as the frequency of the high frequency current injection to be superimposed to the DC bias current of the LD. We have shown that the high frequency injection method could improve the RIN by more than $10 \mathrm{~dB} / \mathrm{Hz}$ at an optimized frequency and modulation depth.

\section{ACKNOWLEDGMENTS}

This work was supported by 'System IC 2010' Project of Korea Ministry of Commerce, Industry and Energy.

*Corresponding author: ymjhon@kist.re.kr

\section{REFERENCES}

[1] G. R. Ray, A. T. Ryan, and G. P. Agrawal, "Control of optical-feedback-induced laser intensity noise in optical data recording," Optical Engineering, 32, 739-45, 1993.

[2] W. W. Chow, S. W. Koch, and M. Sargent III, Semiconductor Laser Physics, Springer-Verlag, Berlin, 1994.

[3] J. C. Yi, and J. Y. Kim, "Investigation of External Feedback Effects on Relative Intensity Noise Characteristics of $405 \mathrm{~nm}$ InAlGaN Laser Diodes," Optical Data Storage, 91-93, 2006. 
[4] J. C. Yi and N. Dagli, "Finite-element analysis of valence band structure and optical properties of quantum-wire arrays on vicinal substrates," IEEE J. Quantum Electronics, 31, pp. 208-18, 1995.

[5] J. Piprek, Semiconductor Optoelectronic Devices, Academic Press, San Diego, pp. 32-48, 2003.

[6] S. Nagahama, T. Yamamoto, M. Sano, and T. Mukai, "Blue-Violet Nitride Laser," Phys. Stat. Sol. (c) 194, no. 2, 423-427, 2002.

[7] S. J. Lee, "Differences in Design Considerations between InGaN and Conventional High-Brightness Light-Emitting Diodes," Journal of the Optical Society of Korea, vol. 2, no. 1, pp. 13-21, 1998.
[8] P. Gallion, F. Jeremier, and J. L. Vey, "Classical optical corpuscular theory of semiconductor laser intensity squeezedlight generation," Optical and Quantum Electronics, 29, 65-70, 1997.

[9] G. P. Agrawal and N. K. Dutta, Long-wavelength Semiconductor Lasers, Van Nostrand Reinhold, NY, 1987.

[10] E. C. Gage and S. Beckens, "Effects of high frequency injection and optical feedback on semiconductor laser performance," SPIE 1316, Optical Data Storage, 199-204, 1990.

[11] Y. Huang, K. Komori, and S. Arai, "Reduction of noise figure in semiconductor laser amplifiers with $\mathrm{Ga}_{1-x} \mathrm{In}_{x} \mathrm{As} /$ GaInAsP/InP strained quantum well structures," IEEE J. Quantum Electron., 29, pp. 2950-6, 1993. 